\title{
Infusion Reactions Related to Infliximab Therapy Are Not Usually Associated with Drug Discontinuation
}


Infliximab has had a major influence on the treatment of a variety of chronic inflammatory diseases since its introduction in the late 1990s and is commonly used in the management of inflammatory arthritis, inflammatory bowel disease (IBD), and psoriasis. Infliximab is administered as an intravenous infusion and, following induction therapy of 3 weight-based doses over 6 weeks, patients receive scheduled therapy every 8 weeks. A major issue for tumor necrosis factor (TNF) inhibitors is their durability. In the major trials of anti-TNF therapy in IBD, only $25 \%$ of initial responders remained in remission after 1 year. This dropoff is mostly due to a combination of secondary loss of response to the medication and adverse effects. Infusion reactions to infliximab can be alarming for patients, physicians, and nurses, and, if misunderstood, may unnecessarily limit further use of the drug. Patients who fail anti-TNF therapy may have limited treatment options, so accurate diagnosis and management of infusion reactions is vital in our attempts to fully optimize these important medications.

Infusion reactions to infliximab are not uncommon, although the true incidence is difficult to estimate due to variations in the reporting of reactions, differing definitions, and issues with retrospectively collected data. In IBD, it is estimated that infusion reactions affect $5 \%-20 \%$ of patients and are typically mild ${ }^{1,2}$. The TREAT registry, a large postmarketing registry of patients with Crohn's disease (CD), reported a $3.1 \%$ reaction rate in 48,279 infliximab infusions. However, reactions severe enough to limit further use of the drug occurred in only $0.07 \%{ }^{3}$.

In this month's issue of The Journal, Kelsall and colleagues report their 8-year experience of infusion reactions in patients with inflammatory arthritis treated at a tertiary referral center in Vancouver, $\mathrm{Canada}^{4}$. Data were available on 4399 infliximab infusions in 200 patients, the majority of whom had rheumatoid arthritis. The authors report an acute infusion reaction rate of $5.8 \%$ and a delayed infusion reaction rate of $0.9 \%$. These data confirm the low rate of infusion reactions to infliximab.

Infusion reactions can be categorized based on their timing (acute or delayed), severity (mild, moderate, or severe), and mechanism (immune or non-immune-mediated). Acute infusion reactions develop within 24 hours of drug delivery although the vast majority occur during infusion. Based on the severity of the symptoms, infusion reactions are also defined as mild, moderate, or severe. The definitions often vary between studies and are typically based on physician assessment. Most reactions are mild or moderate. Several studies have demonstrated that about $3 \%$ of reactions are severe enough to lead to discontinuation of infliximab ${ }^{2,5}$. Acute infusion reactions can be either immune-mediated or non-immune-mediated, and accurately identifying the type of reaction is critical in making future treatment decisions. An immune-mediated acute reaction is a type 1 hypersensitivity (anaphylactic) reaction and is exceptionally rare, and is noted in case reports. Non-immune-mediated acute reactions are far more common and are felt to be related to the rate of the infusion. Type 1 hypersensitivity reactions typically cause dyspnea with wheezing, chest tightness, and hypotension. Bronchospasm is a characteristic symptom of this type of reaction. A true anaphylactic reaction is a contraindication to further use of the drug. Fortunately, the majority of acute infusion reactions are non-immune-mediated and are not a contraindication to ongoing treatment. There is substantial indirect evidence that these reactions are not immune-mediated. Most infusion reactions resolve with discontinuation or slowing of the infusion. Although dyspnea may be significant, there is no associated bronchospasm. The majority of patients can be successfully retreated. Reactions can occur on the first infusion and do not recur with every infusion. Direct evidence for a non-immune-mediated process comes from the Mount

See Infliximab infusion reactions in RA, page 1539

Personal non-commercial use only. The Journal of Rheumatology Copyright (c) 2012. All rights reserved. 
Sinai Medical Center in New York, where researchers studied reactions in 479 infliximab infusions in 165 consecutive patients with IBD. Twenty-nine of 479 (6.1\%) infusions were complicated by a reaction, the majority of which were mild or moderate. In a subanalysis of 14 acute infusion reactions in 11 patients, all had normal tryptase and $\operatorname{IgE}$ levels ${ }^{6}$. A more recent study from Copenhagen evaluated 20 patients with severe acute infusion reactions and found none had anti-infliximab IgE antibodies ${ }^{7}$.

Delayed reactions can occur from 1 to 14 days after infusion, although most reactions occur from 5 to 7 days. These serum-sickness-like reactions may be a type 3 delayed hypersensitivity reaction. They tend to be associated with long treatment intervals between infliximab infusions. Symptoms typically include myalgia, arthralgia, joint pain, and flu-like symptoms, including fever. It is important to differentiate a delayed infusion reaction from drug-induced lupus erythematosus, worsening of the rheumatologic condition, an extraintestinal manifestation of IBD, or a viral syndrome. A true delayed reaction seems to portend a poor prognosis for further infliximab use. In one series of over 600 patients with $C D$ treated with infliximab, delayed infusion reactions occurred in $7 \%$, and over $90 \%$ of these patients discontinued infliximab permanently ${ }^{2}$.

Confirming previous studies, the majority of infusion reactions in the study by Kelsall, et al were acute and of mild or moderate severity. The authors did not differentiate between immune-mediated and non-immune-mediated acute reactions. Infusion reactions, both acute and delayed, were most common during the initial infusions and declined with increasing number of infusions. Pruritis, headache, and facial flushing were among the most common symptoms of acute reactions. Delayed infusion reactions were less common than acute reactions, but were more likely to be moderate in severity. They occurred a mean of 7.2 days after infliximab infusion.

Risk factors for infusion reactions have not been well defined, although it appears that episodic therapy, particularly with a long interval between infusions, increases the risk, and concomitant immunosuppression decreases the risk $^{2,7,8}$. Both these factors influence the development of antibodies to infliximab (ATI). Although the precise clinical relevance of ATI to treatment response and infusion reactions is unknown, they have been associated with infusion reactions in a number of studies ${ }^{9,10}$. In the initial trials of infliximab in $\mathrm{CD}$, acute infusion reactions occurred in $16 \%(42 / 254)$ of infusions in patients positive for ATI compared to $8 \%(55 / 656)$ of infusions in patients negative for ATI $^{1}$. Overall, ATI were associated with a $12 \%$ absolute increase in infusion reactions ${ }^{1}$. Episodic therapy is associated with an increased risk of ATI and with infusion reactions. Fidder, et al demonstrated that an induction regimen and scheduled maintenance therapy were each associated with a decreased risk of infusion reaction $(\mathrm{OR} 0.56)^{2}$. In the same study, concomitant immunomodulation was also associated with a decreased risk of infusion reactions (OR $0.43)^{2}$. This was later confirmed in the randomized, doubleblind, placebo-controlled SONIC trial in CD, which compared infliximab, azathioprine, and combination therapy ${ }^{7}$. Recent data from Denmark showed that episodic dosing was a strong predictor of acute severe infusion reactions (OR 5; 95\% CI 2-13) and combination immunosuppression was associated with a lower risk of infusion reactions compared to infliximab monotherapy (OR $0.3,95 \%$ CI $0.2-0.9)^{7,11}$.

From a clinical viewpoint, the influence of infusion reactions on future infliximab treatments is important. Kelsall, et al did not report their infliximab discontinuation rates $^{4}$, but in a prospective open-label study in RA $(n=113), 2.7 \%$ of the patients discontinued infliximab due to an infusion reaction $^{12}$. In the early studies of infliximab in CD, $2.8 \%$ of patients discontinued the drug due to an infusion reaction ${ }^{1}$. The Mount Sinai study reported a drug-cessation rate of $1.2 \%{ }^{6}$. A number of studies have shown high success rates for reinfusion following a reaction when retreatment protocols utilizing antihistamines, acetaminophen, and corticosteroids are used ${ }^{6,13}$. Infusion centers need to have qualified personnel, equipment, and medications to deal with these potentially severe infusion reactions. As experience using infliximab increases over time, widespread use of maintenance therapy and appropriate management of non-immune-mediated reactions will hopefully lead to lower drug discontinuation rates.

Infliximab remains a valuable drug in the treatment of a variety of chronic inflammatory disorders. Secondary loss of response and discontinuation due to adverse events is common. Optimizing the use of infliximab with adequate dosing and regular maintenance therapy is important to prevent the development of antibodies to the drug and possible infusion reactions. When an infusion reaction does occur, it is important to appropriately characterize it and determine whether one can safely continue the drug. The majority of reactions are non-immune and mild, and do not preclude further infliximab therapy. Use of premedication protocols following infusion reactions has been successful and is associated with a low drug discontinuation rate. For severe infusion reactions, the risks and benefits of further infusion should be weighed against other therapeutic options. True acute, immune-mediated hypersensitivity (anaphylactic) reactions are very rare, but must be recognized as these patients should not be reinfused. Delayed infusion reactions are more likely to be associated with drug discontinuation. Infusion centers should have protocols in place for both the diagnosis and management of infusion reactions to prevent premature discontinuation of infliximab in order to optimize our patients' treatment options. 
GARRETT CULLEN, MD; ADAM S. CHEIFETZ, $M$, Center for Inflammatory Bowel Disease, Division of Gastroenterology, Beth Israel Deaconess Medical Center and Harvard Medical School, Boston, Massachusetts 02215, USA

Address correspondence to Dr. A. Cheifetz, Center for Inflammatory Bowel Disease, Division of Gastroenterology, Beth Israel Deaconess Medical Center, 330 Brookline Ave., Boston, MA 02215.

E-mail: acheifet@bidmc.harvard.edu

\section{REFERENCES}

1. Hanauer SB, Feagan BG, Lichtenstein GR, Mayer LF, Schreiber S, Colombel JF, et al. Maintenance infliximab for Crohn's disease: The ACCENT I randomised trial. Lancet 2002;359:1541-9.

2. Fidder H, Schnitzler F, Ferrante M, Noman M, Katsanos K, Segaert $\mathrm{S}$, et al. Long-term safety of infliximab for the treatment of inflammatory bowel disease: A single-centre cohort study. Gut 2009;58:501-8.

3. Lichtenstein GR, Feagan BG, Cohen RD, Salzberg BA, Diamond $\mathrm{RH}$, Chen DM, et al. Serious infections and mortality in association with therapies for Crohn's disease: TREAT registry. Clin Gastroenterol Hepatol 2006;4:621-30.

4. Kelsall J, Rogers P, Galindo G, De Vera MA. Safety of infliximab treatment in patients with rheumatoid arthritis in a real-world clinical setting: Description and evaluation of infusion reactions. J Rheumatol 2012;39:1539-45.

5. Uthman I, Touma Z, El-Sayyad J, Zaitoun F. Successful retreatment with infliximab in patients with prior severe infusion reactions. Clin Rheumatol 2006;25:540-1.

6. Cheifetz A, Smedley M, Martin S, Reiter M, Leone G, Mayer L, et al. The incidence and management of infusion reactions to infliximab: a large center experience. Am J Gastroenterol 2003;98:1315-24.
7. Steenholdt C, Svenson M, Bendtzen K, Thomsen OO, Brynskov J, Ainsworth MA. Severe infusion reactions to infliximab: aetiology, immunogenicity and risk factors in patients with inflammatory bowel disease. Alimentary pharmacology \& therapeutics;34:51-8.

8. Colombel JF, Sandborn WJ, Reinisch W, Mantzaris GJ, Kornbluth A, Rachmilewitz D, et al. Infliximab, azathioprine, or combination therapy for Crohn's disease. N Engl J Med 2010;362:1383-95.

9. Baert F, Noman M, Vermeire S, Van Assche G, D' Haens G, Carbonez A, et al. Influence of immunogenicity on the long-term efficacy of infliximab in Crohn's disease. N Engl J Med 2003;348:601-8.

10. Farrell RJ, Alsahli M, Jeen YT, Falchuk KR, Peppercorn MA, Michetti P. Intravenous hydrocortisone premedication reduces antibodies to infliximab in Crohn's disease: a randomized controlled trial. Gastroenterology 2003;124:917-24.

11. Moss AC, Fernandez-Becker N, Jo Kim K, Cury D, Cheifetz AS. The impact of infliximab infusion reactions on long-term outcomes in patients with Crohn's disease. Aliment Pharmacol Ther 2008;28:221-7.

12. Wasserman MJ, Weber DA, Guthrie JA, Bykerk VP, Lee P, Keystone EC. Infusion-related reactions to infliximab in patients with rheumatoid arthritis in a clinical practice setting: relationship to dose, antihistamine pretreatment, and infusion number. J Rheumatol 2004;31:1912-7.

13. Lequerre T, Vittecoq O, Klemmer N, Goeb V, Pouplin S, Menard $\mathrm{JF}$, et al. Management of infusion reactions to infliximab in patients with rheumatoid arthritis or spondyloarthritis: experience from an immunotherapy unit of rheumatology. J Rheumatol 2006; 33:1307-14.

J Rheumatol 2012;39:1500-2; doi:10.3899/jrheum.120649 